\title{
Mitochondrial DNA genotyping efficiently reveals clonality of synchronous endometrial and ovarian cancers
}

\author{
Flora Guerra ${ }^{1,8}$, Giulia Girolimetti ${ }^{1,8}$, Anna Myriam Perrone ${ }^{2}$, Martina Procaccini ${ }^{2}$, \\ Ivana Kurelac ${ }^{1}$, Claudio Ceccarelli ${ }^{3}$, Dario De Biase ${ }^{4}$, Giacomo Caprara ${ }^{5}$, Claudio Zamagni ${ }^{6}$, \\ Pierandrea De Iaco $^{2}$, Donatella Santini ${ }^{7}$ and Giuseppe Gasparre ${ }^{1}$ \\ ${ }^{1}$ Department of Medical and Surgical Sciences, Unit of Medical Genetics, University Hospital \\ S.Orsola-Malpighi, Bologna, Italy; ${ }^{2}$ Department of Obstetrics and Gynecology, Oncologic Gynecology Unit, \\ University Hospital S.Orsola-Malpighi, Bologna, Italy; ${ }^{3}$ Department of Experimental, Diagnostic, and Specialty \\ Medicine, University of Bologna, Bologna, Italy; ${ }^{4}$ Laboratory of Molecular Pathology, Anatomic Pathology, \\ Bellaria Hospital, Bologna, Italy; ${ }^{5}$ Unit of Oncology and Transplant Pathology, University Hospital S.Orsola- \\ Malpighi, Bologna, Italy; ${ }^{6}$ Institute of Oncology, Medical Oncology Unit, University Hospital S.Orsola-Malpighi, \\ Bologna, Italy and ${ }^{7}$ Department of Pathology, University Hospital S.Orsola-Malpighi, Bologna, Italy
}

\begin{abstract}
Simultaneous independent primary tumors of the female genital tract occur in 1-2\% of gynecological cancer

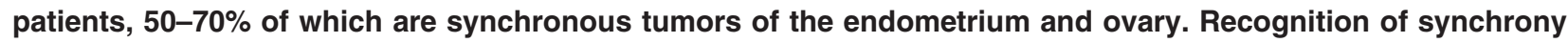
upon multiple tumors is crucial for correct prognosis, therapeutic choice, and patient management. Current guidelines for determining synchrony, based on surgical and histopathological findings, are often ambiguous and may require further molecular analyses. However, because of the uniqueness of each tumor and of its intrinsic heterogeneity, these analyses may sometimes be inconclusive. A role for mitochondrial DNA genotyping was previously demonstrated in the diagnosis of synchronous endometrial and ovarian carcinoma. We have analyzed 11 sample pairs of simultaneously revealed endometrial and ovarian cancers and have thereby applied conventional histopathological criteria, current molecular analyses (microsatellite instability, $\beta$-catenin immunohistochemical staining/CTNNB1 mutation screening), and mitochondrial DNA sequencing to distinguish separate independent tumors from metastases, comparing the performance and the informative potential of such methods. We have demonstrated that in ambiguous interpretations where histopathological criteria and canonical molecular methods fail to be conclusive, mitochondrial DNA analysis may act as a needle of balance and allow to formulate a diagnosis in $45.5 \%$ of our cases. Additional advantages of mitochondrial DNA genotyping, besides the high level of information we demonstrated here, are the easy implementation and the need for small amounts of starting material. Our results show that mitochondrial DNA genotyping may provide a substantial contribution to indisputably recognize the metastatic nature of simultaneously detected endometrial and ovarian cancers and may change the final staging and clinical management of these patients.
\end{abstract}

Modern Pathology (2014) 27, 1412-1420; doi:10.1038/modpathol.2014.39; published online 14 March 2014

Keywords: gynecological cancer; mitochondrial DNA mutations; synchronous tumors

About $1-2 \%$ of women with gynecological cancers are found to have simultaneous independent primary malignancies. Synchronous tumors in the

Correspondence: Dr F Guerra, PhD, Department of Medical and Surgical Sciences, Unit of Medical Genetics - Pad.11, University Hospital S.Orsola-Malpighi, via Massarenti 9, 40138, Bologna, Italy.

E-mail: guerraflora@gmail.com

${ }^{8} \mathrm{Co}$-first authors.

Received 21 November 2013; revised 30 December 2013; accepted 31 December 2013; published online 14 March 2014 ovary and endometrium are the commonest combination (50-70\%) among all synchronous female genital tract malignancies. Only a subset of these can be accurately categorized by standard histological examination. Criteria for synchrony diagnosis were first documented in $1985^{1}$ and subsequently detailed in $1998 .^{2}$ Unfortunately, a relevant fraction of cases remains which may not be classified with confidence, either because of widespread involvement, in which case the distinction is of academic rather than practical or prognostic significance, or, more importantly, because of overlapping or 
ambiguous histological features. Several molecular analyses, such as microsatellite instability, $\beta$-catenin expression, and CTNNB1, TP53, K-RAS, PIK3CA, and PTEN mutation screening are helpful to categorize synchronous ovarian cancer/endometrial cancer in cases where clinical and pathological parameters are inconclusive, albeit with a relatively low sensitivity. ${ }^{3}$ Nonetheless, screening of such a panel of genes may be labor-intensive and expensive, especially as the tumor suppressor genes may acquire inactivating genetic lesions at multiple spots. Recognition of the synchronous or metastatic nature of endometrial and ovarian cancers is of great importance as the correct diagnosis implies different therapeutic strategies, namely chemoradiotherapy for advanced endometrial cancer and poly-chemotherapy for ovarian cancer. ${ }^{4,5} \mathrm{We}$ recently proposed mitochondrial DNA genotyping as a tool to help identify metastatic ovarian/endometrial cancers. ${ }^{6}$ In fact, mitochondrial DNA mutations are extremely common somatic events in human cancers, ${ }^{7}$ as the mitochondrial genome is more susceptible to mutations occurrence than nuclear DNA, and they have been shown to help define progression of the disease, such as in the case of endometrial carcinoma. ${ }^{8}$ Detection of a random somatic mitochondrial DNA mutation in both endometrial and ovarian cancers of the same patient may be considered as a marker of clonality of the two lesions, as it is virtually impossible that the same tumor-specific mutation may arise in two independent neoplasms. ${ }^{9}$

In this work, we tested mitochondrial genome sequencing as an approach to distinguish synchronous vs metastatic ovarian/endometrial cancer showing the informative nature and efficacy of this method.

\section{Material and methods}

\section{Case Series}

The study was performed on 11 cases of simultaneous ovarian and endometrial tumor patients with a mean age of 56 years (range from 40 to 70 years) diagnosed at S.Orsola-Malpighi Hospital, Bologna. Informed consent was obtained in compliance with the Helsinki Declaration. The study was approved by the local ethical committee and internal review board protocols were followed for collection of samples. An alpha-numeric code (from S1 to S11) was assigned to the cases. Tumor stage was determined according to the International Federation of Gynecology and Obstetrics guidelines (FIGO). ${ }^{10}$

\section{Tumors Specimens}

All samples were formalin-fixed and paraffin-embedded. Haematoxylin and eosin sections were reviewed to identify paraffin blocks with tumor areas. Normal specimens were obtained from formalin-fixed and paraffin-embedded nonmetastatic lymph nodes. The histopathological diag- nosis was made according to WHO criteria and the tumors were staged following the American Joint Committee on Cancer (AJCC) and FIGO. ${ }^{10,11}$ Criteria for distinguishing metastatic from independent primary carcinomas were applied. ${ }^{2}$

\section{$\beta$-Catenin Immunohistochemistry}

$\beta$-Catenin localization was observed by immunohistochemistry using a specific antibody (clone 17C2, Novocastra, Newcastle, UK) on formalin-fixed and paraffin-embedded tissue sections. Three patterns of $\beta$-catenin expression were considered: membranous, if the protein was localized exclusively in the cell membrane; nuclear, when expression was observed in the nucleus, and nuclear/membranous when $\beta$-catenin was simultaneously expressed in membranes and nuclei.

\section{CTNNB1 Mutation Screening}

Total DNA was extracted from microdissected formalinfixed and paraffin-embedded tissue sections using a Mammalian Genomic DNA Miniprep Kit (SIGMA Aldrich, St Louis, MO, USA). CTNNB1 mutation screening of exon 3 was performed using fw- $5^{\prime}$-ATGGAACCAGAC AGAAAAGC-3' $3^{\prime}$ and rv-5'-GCTACTTGTTCTTGAGTGAA G-3' primers as previously described. $^{8}$

\section{Microsatellite Instability Analysis}

To investigate tumors microsatellite instability, genotypes were ascertained using 13 polymorphic markers (BAT26, D17S250, TGFBR2, D2S123, D5S346, MybT22, BAT25, D18S58, MT1XT20, BAT40, NR21, NR24, CAT25) in tumor and unaffected tissue ${ }^{12}$. Moreover, three tetranucleotide markers (CSF1PO, D7S820, D18S51) were selected for their high level of polymorphism and low degree of microsatellite instability to confirm that tumor and matching unaffected samples derived from the same individual. Microsatellite instability analysis was performed as previously described. ${ }^{12}$ The tumors were classified as low-microsatellite unstable when $<40 \%$ and at least one marker was unstable. Tumors with $>40 \%$ instability were classified as high-microsatellite unstable. Microsatellitestable cases were defined in the absence of instability for any of the tested loci.

\section{Mitochondrial DNA Sequencing and Analysis}

Sanger sequencing of the whole mitochondrial DNA was performed as previously described. ${ }^{6,8}$ The somatic (informative) nature of mitochondrial mutations was ascertained by sequencing mitochondrial DNA from unaffected tissues. All mitochondrial genome analyses were performed following a quality-check protocol. ${ }^{13,14}$

\section{Fluorescent PCR (F-PCR)}

F-PCR was performed using AmpliTaq Gold polymerase (Applied Biosystems) in presence of a fluorescently 
labeled forward or reverse primer. Primers used for the m.11873insC in MT-ND4 were: fw-5'-GCACTCACAGTCGCATCATAA-3' and rv-5'-(Flc)-TTTGATCAGGAGAACG TGGTT- $3^{\prime}$. Primers used for the m.12425insA in MT-ND5 were: fw-5'-(Flc)-ACCACCCTAACCCTAACTTC-3' and rv-5'-GGCTCAGTGTCAGTTCGAG-3'. PCR products were analyzed following a previously described protocol. ${ }^{15}$

\section{Denaturing High Performance Liquid Chromatography (dHPLC) Analysis}

For the m.1474G $>$ A/(MT-12S), PCR was performed using AmpliTaq Gold polymerase (Applied Biosystems) and fw-5'-GCAAACCCTGATGAAGGCTA-3' and rv-5'-ACTCT GGTTCGTCCAAGTGC-3' ${ }^{\prime}$ primers. The amplification product was analyzed by WAVE Nucleic Acid Fragment Analysis System (Transgenomic, Omaha, NE, USA) using $60.2^{\circ} \mathrm{C}$ separation temperature. Data analysis was performed as previously described. ${ }^{15,16}$

\section{Results}

\section{Histopathological Diagnosis}

Based on histopathological analyses, five out of eleven $(45.5 \%)$ cases were diagnosed as suspected synchronous tumors (S1, S5, S7, S9, and S11), other five $(45.5 \%)$ cases as suspected metastatic tumors (S3, S4, S6, S8 and S10) and for one case (9\%) diagnosis was not possible (S2) (Table 1).

\section{Molecular Standard Criteria for Synchrony Diagnosis}

Nuclear localization of $\beta$-catenin and/or detection of CTNNB1 mutations are known to be more likely associated with independent primary endometrial and ovarian cancer, whereas membranous pattern of $\beta$-catenin and absence of CTTNB1 mutations commonly occur in metastatic tumors. ${ }^{17}$ We have thus performed $\beta$-catenin immunohistochemistry and CTNNB1 sequencing. Cases S3, S4, S7, S10, and S11 showed membranous $\beta$-catenin localization in both ovarian and endometrial cancer, while cases S8 and S9 displayed heterogeneous nuclear/membranous $\beta$-catenin expression in both neoplasms. Cases S5 and S6 presented membranous $\beta$-catenin localization in ovarian cancer and heterogeneous nuclear/ membranous $\beta$-catenin expression in endometrial cancer. Cases S1 and S2 displayed heterogeneous nuclear/membranous $\beta$-catenin localization in ovarian cancer and homogeneous membranous and nuclear $\beta$-catenin expression in endometrial cancer, respectively (Table 2). Based on the identical and homogeneous $\beta$-catenin localization in endometrial and ovarian cancer, ${ }^{3,17}$ S3, S4, S7, S10, and S11 appeared to be metastatic tumors. In S7 (Figure 1) and S11, $\beta$-catenin-based immunohistochemistry diagnosis was in contrast with the histopathological conclusion of synchrony. For the other cases, because of the heterogeneous $\beta$-catenin localiza-
Table 1 Pathological stage based on Scully's criteria ${ }^{2}$

\begin{tabular}{|c|c|c|c|c|c|c|}
\hline Patients & Age & $\begin{array}{l}\text { OC FIGO } \\
\text { stage }\end{array}$ & $\begin{array}{c}\text { EC FIGO } \\
\text { stage }\end{array}$ & $\begin{array}{c}O C \\
\text { histology }\end{array}$ & $\begin{array}{c}E C \\
\text { histology }\end{array}$ & $\begin{array}{l}S / M \text { (final } \\
\text { diagnosis) }\end{array}$ \\
\hline S1 & 50 & IIIC & IIA & E G2 & E G2 & S \\
\hline S2 & 57 & IIIB & IB & U G3 & U G3 & UN \\
\hline S3 & 70 & - & IIIC & U G3 & U G3 & M (primary EC) \\
\hline S4 & 52 & - & IIA & CC & CC & $\mathrm{M}$ (primary EC) \\
\hline S5 & 58 & IC & IB & E G1 & E G1 & S \\
\hline S6 & 59 & - & IIIC & SP G3 & SP G3 & M (primary EC) \\
\hline S7 & 54 & IB & IB & E G1 & E G1 & $\mathrm{S}$ \\
\hline S8 & 55 & IIIB & - & E G2 & E G2 & $\mathrm{M}($ primary OC) \\
\hline S9 & 49 & IC & IB & M & E G2 & S \\
\hline S10 & 70 & IIIC & - & CC & CC & $\mathrm{M}$ (primary OC) \\
\hline S11 & 40 & IIC & IB & SP & SP & S \\
\hline
\end{tabular}

Abbreviations: CC, clear cells; EC, endometrial cancer; E, endometrioid; G, grade; M, metastatic; M, mucinous; OC, ovarian cancer; S, synchronous; SP, serous papillary; U, undifferentiated.

tion, this analysis was not informative. Therefore, $\beta$-catenin immunohistochemistry was helpful for diagnosis in five out of eleven (45.5\%) cases. Upon correlation of $\beta$-catenin immunohistochemistry patterns with CTNNB1 mutations, we observed that in S3, S4, S7, S10, and S11 the membranous pattern was associated with absence of CTTNB1 mutations and with diagnosis of metastases. We detected tumor-specific CTNNB1 mutations in S1, S2, S8, and S9, where $\beta$-catenin localization was heterogeneous in one or both tumors. In particular, S1 was shown to harbor the c.100G $>$ A mutation in endometrial and ovarian tumors, ${ }^{18}$ in $\mathrm{S} 8$ the same c.101G $>$ A mutation was found in both tumors, while S9 ovarian/endometrial cancers harbored a novel c.104T $>$ G mutation. These cases could not be associated with independent primary endometrial and ovarian cancers since a clear $\beta$-catenin nuclear pattern was not found and the same somatic mutation was identified in both endometrial and ovarian neoplasias (Table 2). In S2, the c.101G $>$ A mutation was found only in endometrial cancer in association with a homogeneous nuclear $\beta$-catenin localization, thereby synchrony was hypothesized. In conclusion, combination of the two methods was informative in six out of eleven (54.5\%) cases.

We next performed microsatellite instability analysis, as this technique was shown to add informativity for diagnosis of simultaneously detected endometrial/ovarian cancers. ${ }^{3}$ Microsatellite instability analysis revealed nine out of eleven (82\%) microsatellite-stable samples and two out of eleven $(18 \%)$ unstable samples (S2 and S11). Case S2 ovarian cancer was defined as highly microsatellite unstable since six out of thirteen (46\%) microsatellites analyzed were found unstable (BAT25, MtlxT20, BAT40 (Figure 2a, red arrows), BAT26, NR21, CAT25), while S2 endometrial cancer was defined as low microsatellite unstable as only NR24 $(1 / 13,8 \%)$ showed instability (Figure 2a). This molecular analysis allowed us to hypothesize a synchronous origin of these two tumors, which further reinforced the diagnosis based on $\beta$-catenin 
Table 2 Comparison between histopathological, molecular, and mitochondrial DNA diagnosis

\begin{tabular}{|c|c|c|c|c|c|c|c|c|c|c|}
\hline Samples & Tissue & $\begin{array}{c}\text { Histopatological } \\
\text { diagnosis }\end{array}$ & $\begin{array}{c}\beta \text {-Catenin } \\
\text { localization }\end{array}$ & $\begin{array}{l}\text { CTNNB1 } \\
\text { mutations }\end{array}$ & $M S I$ & $\begin{array}{l}\text { Molecular } \\
\text { diagnosis }\end{array}$ & $\begin{array}{l}\text { MtDNA } \\
\text { mutations }\end{array}$ & Gene & Het & $\begin{array}{l}\text { Mitochondrial } \\
\text { DNA diagnosis }\end{array}$ \\
\hline S1 & $\begin{array}{l}\text { EC } \\
\text { OC }\end{array}$ & $S$ & $\begin{array}{c}\text { M } \\
\text { NM }\end{array}$ & c. $100 \mathrm{G}>\mathrm{A}$ & - & UN & $\begin{array}{c}\text { m.11873insC } \\
\text { m.15247C }>\text { T } \\
\text { m.2680T }>C\end{array}$ & $\begin{array}{c}M T-N D 4 \\
M T-C Y B \\
M T- \\
\text { RNR2 }\end{array}$ & $\begin{array}{c}\mathrm{Y} \\
\text { No } \\
\mathrm{Y}\end{array}$ & M \\
\hline S2 & $\begin{array}{l}\text { EC } \\
\text { OC }\end{array}$ & UN & $\begin{array}{c}\mathrm{N} \\
\mathrm{NM}\end{array}$ & $\begin{array}{c}\text { c. } 101 \mathrm{G}>\mathrm{A} \\
-\end{array}$ & $\begin{array}{l}\text { MSI-L } \\
\text { MSI-H }\end{array}$ & S & - & - & & UN \\
\hline S3 & $\begin{array}{l}\text { EC } \\
\text { OC }\end{array}$ & M & $\begin{array}{l}\mathrm{M} \\
\mathrm{M}\end{array}$ & - & - & M & m.12425insA & MT-ND5 & $\mathrm{Y}$ & M \\
\hline S4 & $\begin{array}{l}\mathrm{EC} \\
\mathrm{OC}\end{array}$ & M & $\begin{array}{l}M \\
M\end{array}$ & - & - & M & $\begin{array}{l}\mathrm{m} .5567 \mathrm{~T}>\mathrm{C} \\
\mathrm{m} .13994 \mathrm{~T}>\mathrm{C}\end{array}$ & $\begin{array}{l}M T-T W \\
M T-N D 5\end{array}$ & $\begin{array}{l}\mathrm{Y} \\
\mathrm{Y}\end{array}$ & M \\
\hline S5 & $\begin{array}{l}\text { EC } \\
\text { OC }\end{array}$ & S & $\begin{array}{c}\mathrm{NM} \\
\mathrm{M}\end{array}$ & - & - & UN & - & - & & UN \\
\hline S6 & $\begin{array}{l}\mathrm{EC} \\
\mathrm{OC}\end{array}$ & M & $\begin{array}{c}\mathrm{NM} \\
\mathrm{M}\end{array}$ & - & - & UN & - & - & & UN \\
\hline S7 & $\begin{array}{l}\text { EC } \\
\text { OC }\end{array}$ & S & $\begin{array}{l}M \\
M\end{array}$ & - & - & M & - & - & & UN \\
\hline S8 & $\begin{array}{l}\text { EC } \\
\text { OC }\end{array}$ & M & $\begin{array}{l}\text { NM } \\
\text { NM }\end{array}$ & c. $101 \mathrm{G}>\mathrm{A}$ & - & UN & - & - & & UN \\
\hline S9 & EC & S & NM & c. $104 \mathrm{~T}>\mathrm{G}$ & - & UN & m.1474G $>$ A & $\begin{array}{c}M T- \\
R N R 1 \\
M T-C Y B\end{array}$ & $\mathrm{Y}$ & M \\
\hline S10 & $\begin{array}{l}\text { OC } \\
\text { OC }\end{array}$ & M & $\begin{array}{l}\mathrm{NM} \\
\mathrm{M} \\
\mathrm{M}\end{array}$ & - & - & M & $\begin{array}{c}\mathrm{m} .15573 \mathrm{~T}>\mathrm{C} \\
-\end{array}$ & $\begin{array}{c}M T-C Y B \\
-\end{array}$ & $Y$ & UN \\
\hline S11 & $\begin{array}{l}\text { EC } \\
\text { OC }\end{array}$ & S & $\begin{array}{l}\mathrm{M} \\
\mathrm{M}\end{array}$ & - & $\mathrm{LOH}$ & M & m. $4722 \mathrm{~A}>\mathrm{G}$ & $M T-N D 2$ & $\mathrm{Y}$ & M \\
\hline
\end{tabular}

Abbreviations: AA, amino acid; EC, endometrial cancer; Het, heteroplasmy; LOH, loss of heterozygosity; M, membranous; M, metastatic; MSI, microsatellite instability; N, nuclear; NM, nuclear/membranous; OC, ovarian Cancer; S, synchronous; UN, undefined; Y, yes.

localization and CTNNB1 mutation screening. Conversely, S11 ovarian and endometrial cancers showed to share the LOH (loss of heterozygosity) at D17S250 (17q11.2/BRCA1), which, along with the matching $\beta$-catenin localization observed in this case, allowed us to identify $\mathrm{S} 11$ as metastatic (Figure 2b), which was in disagreement with the histopathology-based diagnosis.

\section{MtDNA Augments Informativity and Reinforces Diagnosis}

With the aim to increase the percentage of cases for which an unequivocal diagnosis could be provided, we next implemented mitochondrial DNA sequencing, an easy to perform and standardized approach. The entire sequence of mitochondrial genome was obtained from all 22 single ovarian and endometrial cancer samples. For all 11 patients, DNA extracted from unaffected tissue was available and used to detect tumor-specific and nontumor-specific variants. Overall, 11 tumor-specific variants were found in five out of eleven patients (45.5\%) (Table 2).

We found the m.11873insC/(MT-ND4), the $\mathrm{m} .15247 \mathrm{C}>\mathrm{T} /(M T-C Y B)$, and the m.2680T $>\mathrm{C} /$ (MT-RNR2) in both OC and EC of S1 (Figure 3a); the m.12425insA/(MT-ND5) in both tumors of S3 (Figure 3c); the m.5567T $>C /(M T-T W$ ) and the $\mathrm{m} .13994 \mathrm{~T}>\mathrm{C} /(M T-N D 5)$ in both endometrial and ovarian neoplasia of S4 (Figure $3 \mathrm{~b}$ ), which also carried the m.15172G $>\mathrm{A} /(M T-C Y B)$ only in endometrial cancer. In S9 the m.1474G $>A /(M T-R N R 1)$ and the $\mathrm{m} .15573 \mathrm{~T}>\mathrm{C} /(M T-C Y B)$ were found in both tumors whereas the m.531T $>\mathrm{C} /(M T-D L O O P)$ only in ovarian cancer (Figure 3d). Finally, S11 carried the m.4722A $>\mathrm{G} /(M T-N D 2)$ in both endometrial and ovarian cancer (Figure 3e). None of the corresponding matched nontumor samples was shown to carry these mitochondrial DNA mutations.

Owing to the mitochondrial genome physiological polyploidy, mitochondrial DNA mutations may be present in homo/heteroplasmy. Given the significance of tumor-specific mitochondrial DNA mutations for the identification of a clonal origin in suspected synchronous tumors, a precise quantification of the mitochondrial DNA mutation load and efficient detection of low-level heteroplasmies are of paramount importance. Indeed, identification of low-heteroplasmic germline mitochondrial DNA mutations in matched nontumor samples might render some mutations noninformative. Therefore, in order to establish the tumor specificity and the heteroplasmy level of the mutations found in S1, S3, and S9, and to confirm efficiency of mitochondrial DNA sequencing in synchrony diagnosis, we used F-PCR and dHPLC, both methods shown to be sensitive enough to detect heteroplasmy levels as low as $2 \% .^{14}$

In patients $\mathrm{S} 1$ and $\mathrm{S} 3$, where we respectively detected the m.11873insC/(MT-ND4) and the m.12425insA/ (MT-ND5), the degree of heteroplasmy was tested by 


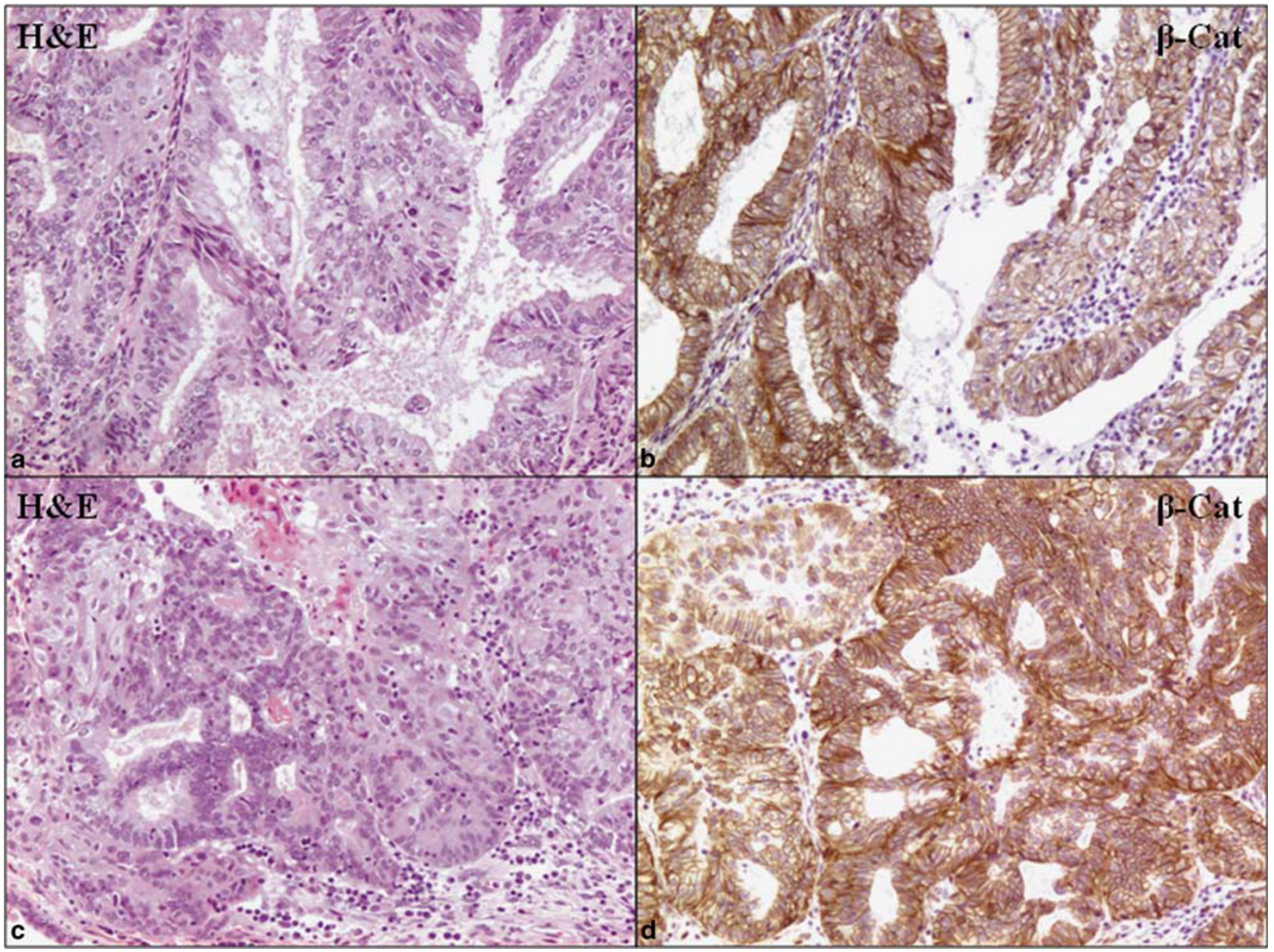

Figure 1 Histopathological diagnosis vs canonical $\beta$-catenin immunohistochemistry in a representative case (S7): haematoxylin and eosin sections showed synchronous endometrioid adenocarcinoma of the endometrium (a) and ovary (c), while $\beta$-catenin immunohistochemistry showed a prevalent membranous pattern in both tumors (b, d).

F-PCR. In S1, the analysis revealed two to four C insertions at different heteroplasmy percentages in ovarian cancer and two to seven $\mathrm{C}$ base insertions at different heteroplasmy percentages in endometrial cancer, while nontumor tissue presented no insertions (Figure 4a). We performed the same analysis for case S3 and found a two-A base insertion at the m.12425 in both ovarian and endometrial cancers that was absent in nontumor tissue (data not shown). Likewise, lowlevel germline heteroplasmy was absent from nontumor tissue of case S9, in which the m.1474G $>$ A/ (MT-RNR1) was shown to be present exclusively in ovarian and endometrial cancer samples (Figure 4b). We therefore confirmed the nongermline nature of mutations found in S1, S3, and S9.

We concluded that patients in whom tumorspecific mitochondrial DNA mutations were shared between ovarian and endometrial cancer (S1, S3, S4, S9, and S11) were affected by a metastatic disease, whereas nontumor-specific mutations were not informative for diagnosis. In contrast to independent histopathological diagnosis, mitochondrial
DNA sequencing inferred clonal origin of ovarian and endometrial cancer in cases S1, S9, and S11. In case S11, mitochondrial genome analysis was in agreement with microsatellite instability analysis, defining metastatic cancer diagnosis.

\section{Discussion}

A variety of tumors involving the female genital tract appears simultaneously but independently. It is of paramount importance for pathologists to recognize whether endometrial and ovarian cancers occur as metastatic or unlinked events, as both the prognostic implications and the patient management are largely different. Treatment of ovarian and endometrial tumors when they metastasize in endometrium or ovary requires the use of different pharmacological and surgical approaches, respectively. ${ }^{4,5}$

In this work, we analyzed 11 sample pairs of simultaneously revealed endometrial/ovarian cancers and applied conventional histopathological criteria, 
a
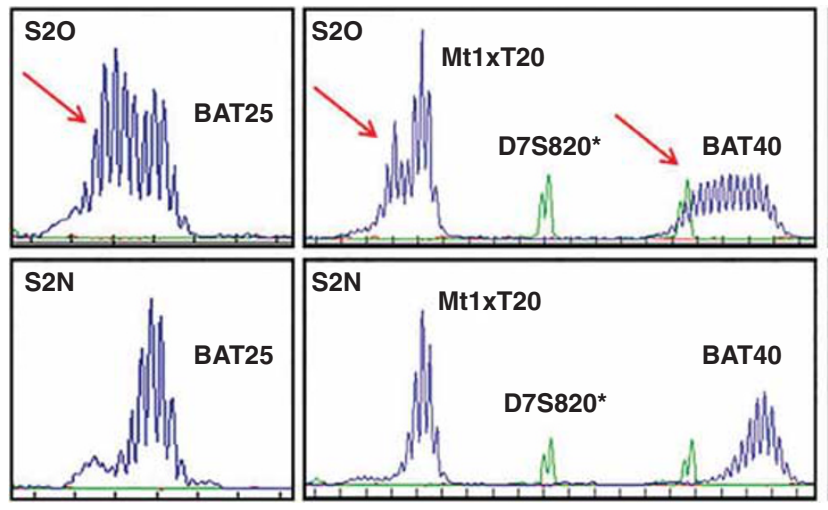

b

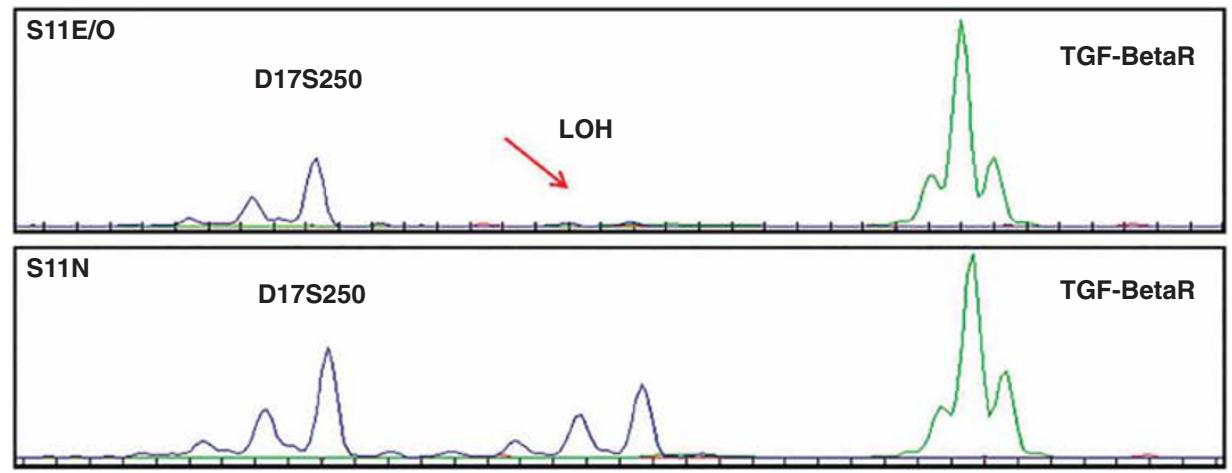

Figure 2 Microsatellite instability analysis. (a) BAT25, Mt1xT20, BAT40, and NR24 microsatellites in S2 endometrial/ovarian cancer compared with matched normal tissue. (b) LOH identified in both endometrial/ovarian cancer samples of S11.

a

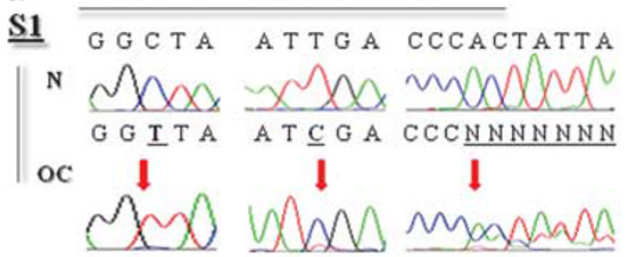

EC

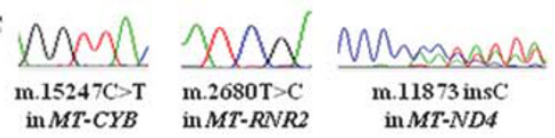

C

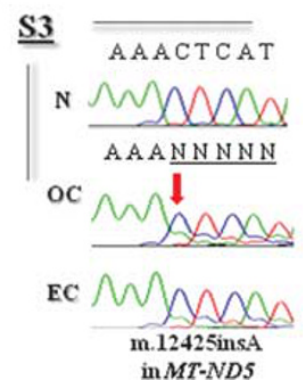

b

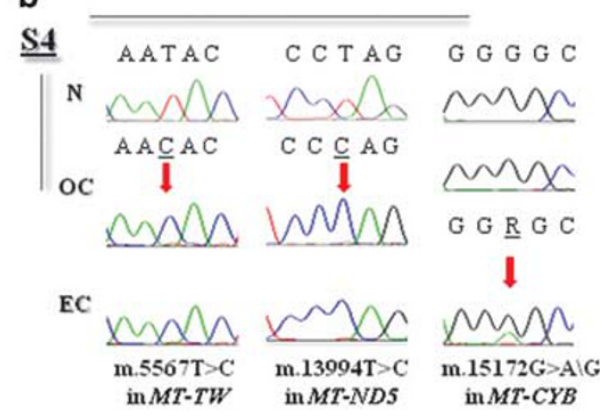

e

Figure 3 Mitochondrial DNA genotyping. Electropherograms of mitochondrial loci harbouring mutations in endometrial/ovarian cancer samples. Red arrows indicate the mutated bases. 
a
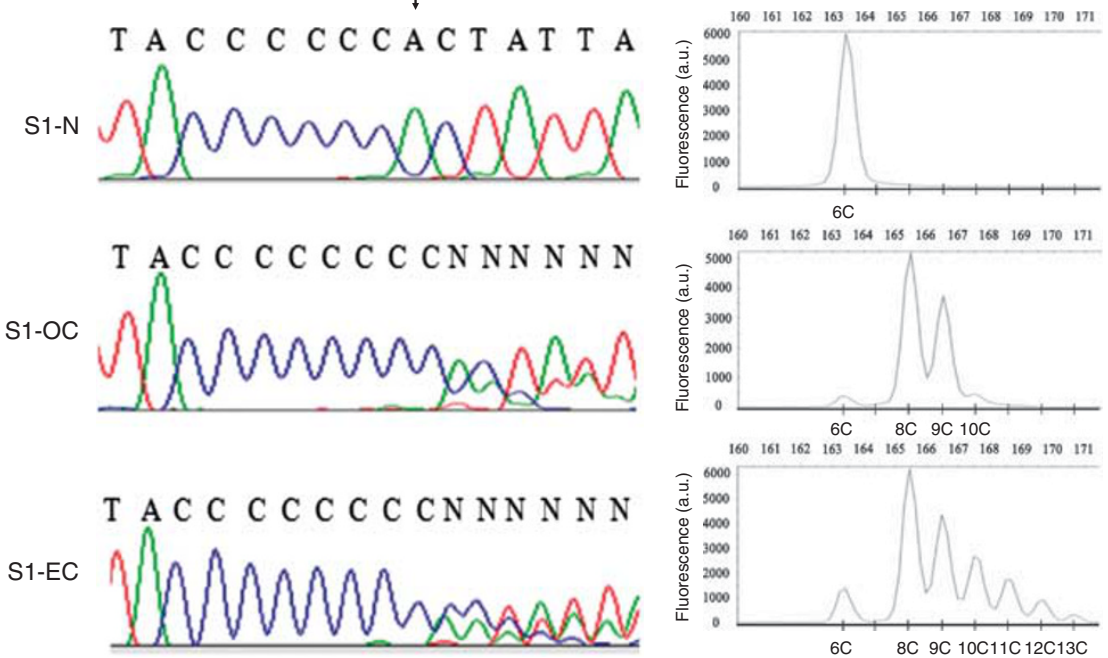

m.11873 insC/(MT-ND4)

b
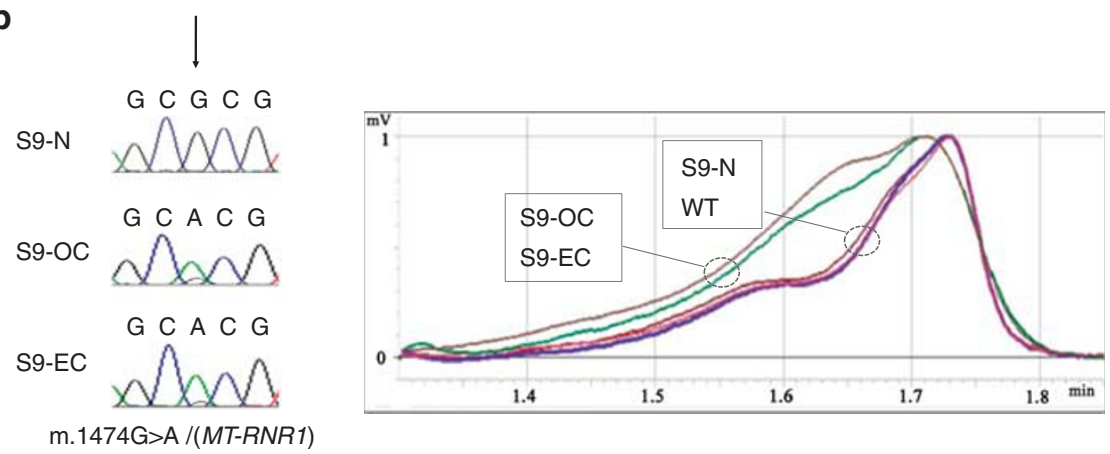

Figure 4 In-depth investigation of the mitochondrial DNA mutation somatic nature (a) electropherogram of the m.11873insC/MT-ND4 in S1. F-PCR analysis in S1 shows two to four inserted cytosines at different heteroplasmy percentages in ovarian cancer (a) and two to seven $\mathrm{C}$ bases insertions at different heteroplasmy percentages in endometrial cancer in the same position (b) compared with nontumor tissue that presents no insertion (c). The arrows indicate the wild-type peaks. (b) Electropherogram of the heteroplasmic m.1474G $>\mathrm{A} /$ MT-RNR1 in ovarian cancer and endometrial cancer in case S9; DHPLC analysis of the m.1474G $>$ A/MT-RNR1. Homo and heteroduplexes are distinguished based on different retention times. Two elution curves for endometrial and ovarian cancer (heteroduplex and homoduplex) and a single elution curve for nontumor tissue and two wild-type controls are present. Wild-type (pink and red), nontumor tissue (purple), endometrial cancer (green), ovarian tumor (brown).

current diagnostic molecular tools, and mitochondrial genome sequencing to achieve a diagnosis of either separate independent tumors or metastases, comparing the performance and the informative potential of such methods.

Histopathological criteria are indisputably those primarily used to reach a conclusion, ${ }^{1-3}$ albeit owing to ambiguous features, cases may occur in which it is not possible to rule out a synchronous or a metastatic nature of the neoplasms, as S2 in our sample set. Such a relative limitation is provided by the fact that application of histopathological criteria is an empiric tool based on the personal experience of the pathologist in charge, and must take into account the heterogeneity of the samples analyzed. In ambiguous cases application of ancillary techniques is mandatory. ${ }^{3}$ Among the molecular methods available, here we have implemented the currently most accepted analyses concerning genetics alterations frequently encountered in single endometrial and ovarian cancers (microsatellite instability, $\beta$-catenin expression, and CTNNB1 mutations). ${ }^{19,20}$

It is striking that microsatellite instability analysis alone was informative only in two $(18 \%)$ cases in our sample set. It was confirmatory in one case and helped define diagnosis in the unclassified S2 case. Overall, informativity was relatively low, especially compared with mitochondrial DNA genotyping, which was used subsequently.

The combination of $\beta$-catenin expression and CTNNB1 mutation screening provided an indication for diagnosis in half our cases. Nevertheless, this analysis failed to provide an unequivocal diagnostic parameter as $\beta$-catenin immunohistochemistry is often heterogeneous and therefore independent or metastatic tumor classification is not without difficulties. Moreover, it is known that independent 
primary tumors may display highly similar immunohistochemistry profiles. ${ }^{3}$ Analogously, it ought to be considered that mutation screening of CTNNB1 may be a relatively reliable criterion to reach a synchrony diagnosis, as virtually all CTNNB1 mutations identified in human tumors, endometrial and ovarian cancer included, are located in exon 3, making this a hot spot for mutation activation of $\beta$-catenin. ${ }^{21}$ The very nature of an oncogene implies that only a few protein residues need to be mutated for its constitutive activation, which is the case for $\beta$-catenin. Hence, the probability that two independent tumors within the same individual may harbor the same mutation by chance is more likely than what would occur for an inactivating mutation in a tumor suppressor gene, especially if activation of that oncogene is required to drive tumorigenesis. Additionally, identical gene mutations may be detected in clearly independent primaries resulting from a 'field effect' of a common oncogenic stimulus ${ }^{17}$ and the finding of different genetic abnormalities, both in terms of subcellular localization and mutation occurrence, may reflect tumor heterogeneity rather than evidence of separate primaries. ${ }^{22}$

In this frame, mitochondrial DNA genotyping displays advantages when the same somatic mutation is found in both endometrial and ovarian cancer. The current variability estimates for mitochondrial genome positions show that a great part of over 16500 nucleotides by which the human mitochondrial DNA is composed varies among individuals with different frequency, as it is reported in human mitochondrial databases. ${ }^{23}$ Moreover, it is ascertained that cancer cells may easily withstand otherwise infrequent pathogenic mutations that affect the respiratory chain, as oxidative metabolism is often shut off in tumors in favor of the Warburg effect. ${ }^{7,24}$ It is therefore reasonable to assume that two independent tumors arising in a single individual may not acquire the same somatic mitochondrial DNA genotype, especially as no 'field effects' are known nowadays. This assumption was the basis of our work, as the occurrence of the same, truly somatic (ie, cancer-specific) mutation detected in both endometrial and ovarian cancer, ought to be an unequivocal marker of a common, clonal origin of both cancers, most likely one being a metastasis of the other. Interestingly, although our sample set was relatively small owing to the rare occurrence of simultaneous endometrial/ovarian cancer, mitochondrial DNA screening revealed to be informative in nearly half of the cases $(45.5 \%)$, a percentage comparable with that of other less precise molecular analyses such as $\beta$-catenin. Such analyses were corroborated and strengthened by the in-depth investigation of the somatic nature of detected mitochondrial DNA mutations to ascertain informativity.

It is worth noting that mitochondrial DNA genotyping was suggestive of a metastatic nature for cases S1, S9, and S11, in disagreement with the independent histopathological diagnosis, while confirming diagnosis of metastatic tumors in cases S3 and S4. In case S11, the detection of LOH trough microsatellite instability analysis supported the result obtained by mitochondrial DNA sequencing, suggesting that novel molecular tools need be implemented to help the pathologist achieve a correct diagnosis, which may not always be the one based merely on histopathological criteria. In cases S1 and S9 no other technique could ascertain whether mitochondrial DNA genotyping or histopathological diagnosis was correct, albeit it appears unlikely that the same mitochondrial mutation may have occurred independently in endometrial and ovarian cancers. Expensive investigations are warranted in this case to unequivocally prove the clonal nature of these neoplasms, such as for instance array-based Comparative Genomic Hybridization. ${ }^{6}$

Overall, in ambiguous interpretations where histopathological criteria and canonical molecular methods fail to be conclusive, mitochondrial DNA analysis may act as a needle of balance and allow to express a correct diagnosis. Advantages of mitochondrial DNA genotyping, besides the high level of informativity we demonstrated here, are the easy implementation, the need for small amounts of DNA, and the relatively low costs (nowadays $<50$ euros per genome), which are bound to become lower with the advent of targeted deep sequencing techniques. Moreover, mitochondrial DNA screening may be performed using a standardized sequencing kit that requires a single operator.

In conclusion, compared with the use of current standard molecular techniques, mitochondrial DNA genotyping revealed to be a robust method for endometrial/ovarian cancer metastasis diagnosis, increasing informativity in cases defined ambiguous by other methods. Therefore, we propose the use of mitochondrial DNA genotyping to be implemented in diagnostic procedures to resolve ambiguous cases of synchronous gynecological malignancies.

\section{Acknowledgments}

We thank M Natale (MS) for technical help. This work has been supported by Grant IG8810 from the Associazione Italiana Ricerca sul Cancro (AIRC), by Grant DISCO-TRIP from Fondazione Umberto Veronesi, and by Grant FIRB 'Futuro in Ricerca' J31J10000040001 to GG; FG is supported by an AIRC biennial fellowship 'Maria Antonietta Carluccio'. IK is supported by an AIRC triennial fellowship 'Borromeo'.

\section{Disclosure/conflict of interest}

The authors declare no conflict of interest. 


\section{References}

1 Ulbright TM, Roth LM. Metastatic and independent cancers of the endometrium and ovary: a clinicopathologic study of 34 cases. Hum Pathol 1985;16:28-34.

2 Scully RE YR, Clement PB. Atlas of Tumor Pathology: Tumors of the Ovary, Maldeveloped Gonads, Fallopian Tube and Broad Ligament 1998;27-50.

3 Singh N. Synchronous tumours of the female genital tract. Histopathology 2010;56:277-285.

4 Bookman MA. First-line chemotherapy in epithelial ovarian cancer. Clin Obstet Gynecol 2012;55:96-113.

5 Hill EK, Dizon DS. Medical therapy of endometrial cancer: current status and promising novel treatments. Drugs 2012;72:705-713.

6 Guerra F, Kurelac I, Magini P, et al. Mitochondrial DNA genotyping reveals synchronous nature of simultaneously detected endometrial and ovarian cancers. Gynecol Oncol 2011;122:457-458.

7 Brandon M, Baldi P, Wallace DC. Mitochondrial mutations in cancer. Oncogene 2006;25:4647-4662.

8 Guerra F, Kurelac I, Cormio A, et al. Placing mitochondrial DNA mutations within the progression model of type I endometrial carcinoma. Hum Mol Genet 2011;20:2394-2405.

9 Foschini MP, Morandi L, Marchetti C, et al. Cancerization of cutaneous flap reconstruction for oral squamous cell carcinoma: report of three cases studied with the mtDNA D-loop sequence analysis. Histopathology 2011;58:361-367.

10 Shepherd JH. Revised FIGO staging for gynaecological cancer. Br J Obstet Gynaecol 1989;96:889-892.

11 Sobin LH, Wittekind Ch (eds). UICC (International Union Against Cancer). TNM classification of malignant tumours, 6th ed. Wiley-Liss: New York, Chichester, Weinheim, Brisbane, Singapore, Toronto, 2002.

12 Morandi L, de Biase D, Visani M, et al. T([20]) repeat in the 3'-untranslated region of the MT1X gene: a marker with high sensitivity and specificity to detect microsatellite instability in colorectal cancer. Int J Colorectal Dis 2012;27:647-656.

13 Kurelac I, MacKay A, Lambros MB, et al. Somatic complex I disruptive mitochondrial DNA mutations are modifiers of tumorigenesis that correlate with low genomic instability in pituitary adenomas. Hum Mol Genet 2013;22:226-238.

14 Porcelli AM, Ghelli A, Ceccarelli C, et al. The genetic and metabolic signature of oncocytic transformation implicates HIF1alpha destabilization. Hum Mol Genet 2010;19:1019-1032.

15 Kurelac I, Lang M, Zuntini R, et al. Searching for a needle in the haystack: comparing six methods to evaluate heteroplasmy in difficult sequence context. Biotechnol Adv 2012;30:363-371.

16 Frueh FW, Noyer-Weidner M. The use of denaturing high-performance liquid chromatography (DHPLC) for the analysis of genetic variations: impact for diagnostics and pharmacogenetics. Clin Chem Lab Med 2003;41:452-461.

17 Irving JA, Catasus L, Gallardo A, et al. Synchronous endometrioid carcinomas of the uterine corpus and ovary: alterations in the beta-catenin (CTNNB1) pathway are associated with independent primary tumors and favorable prognosis. Hum Pathol 2005;36:605-619.

18 Silva R, Marie SK, Uno M, et al. CTNNB1, AXIN1 and APC expression analysis of different medulloblastoma variants. Clinics 2013;68:167-172.

19 Hecht JL, Mutter GL. Molecular and pathologic aspects of endometrial carcinogenesis. J Clin Oncol 2006;24:4783-4791.

20 Kurman RJ, Shih IeM. Pathogenesis of ovarian cancer: lessons from morphology and molecular biology and their clinical implications. Int J Gynecol Pathol 2008; 27:151-160.

21 Ilyas M. Wnt signalling and the mechanistic basis of tumour development. J Pathol 2005;205:130-144.

22 Chang KH, Albarracin C, Luthra R, et al. Discordant genetic changes in ovarian and endometrial endometrioid carcinomas: a potential pitfall in molecular diagnosis. Int J Gynecol Cancer 2006;16:178-182.

23 Rubino F, Piredda R, Calabrese FM, et al. HmtDB, a genomic resource for mitochondrion-based human variability studies. Nucleic Acids Res 2012;40: Database issue D1150-D1159.

24 Iommarini L, Calvaruso MA, Kurelac I, Gasparre G, Porcelli AM. Complex I impairment in mitochondrial diseases and cancer: parallel roads leading to different outcomes. Int J Biochem Cell Biol 2012;45:47-63. 